\title{
Uncertainty Analysis for Natural Gas Transport Pipeline Network Layout: A New Methodology Based on Monte Carlo Method
}

\author{
Jun Zeng $\mathbb{D}^{1},{ }^{1,2}$ Chaoxu Sun $\mathbb{D}^{\mathbb{D}},{ }^{3}$ Zhenjun Zhu $\mathbb{D}^{\mathbb{D}},{ }^{1,4}$ Jiangling $W u^{5}$ and Hongsheng Chen ${ }^{6}$ \\ ${ }^{1}$ School of Transportation, Southeast University, Nanjing 211189, China \\ ${ }^{2}$ Department of Civil, Architectural and Environmental Engineering, The University of Texas at Austin, Austin, TX 78712, USA \\ ${ }^{3}$ Zhejiang Provincial Natural Gas Development Co. Ltd., Hangzhou 310052, China \\ ${ }^{4}$ Department of City and Regional Planning, University of California, Berkeley, Berkeley, CA 94720, USA \\ ${ }^{5}$ School of Civil Engineering and Architecture, Henan University, Kaifeng 475004, China \\ ${ }^{6}$ School of Architecture, Southeast University, Nanjing 210096, China
}

Correspondence should be addressed to Zhenjun Zhu; shangjingqu@163.com

Received 2 November 2017; Revised 6 April 2018; Accepted 10 April 2018; Published 23 May 2018

Academic Editor: Zhi-Chun Li

Copyright (C) 2018 Jun Zeng et al. This is an open access article distributed under the Creative Commons Attribution License, which permits unrestricted use, distribution, and reproduction in any medium, provided the original work is properly cited.

\begin{abstract}
Natural gas plays an increasing important role in the China's energy revolution. The rapid market development and refined government regulation demand improvements in the natural gas transport pipeline network. Therefore, it is of great theoretical and practical significance to conduct a study regarding the layout of pipeline networks. To reflect the comprehensive benefits of pipeline projects and obtain global optimal solution, this study introduces the dominance degree model (DDM). Aiming at optimizing the layout of natural gas transport pipeline networks, this paper studies the uncertainty of the DDM and the corresponding method for network layout. This study proposes an uncertainty analysis based on the Monte Carlo method to quantify the uncertainty of the DDM and its influential factors. Finally, the methodology is applied to the real case of a natural gas transport pipeline project in Zhejiang Province, China. The calculation results suggest that the methodology appropriately addresses the problem of pipeline network layout for natural gas transport. This has important implications for other potential pipeline networks not only in the Zhejiang Province but also throughout China and beyond.
\end{abstract}

\section{Introduction}

Natural gas is an efficient and clean energy source that can be utilised in the production of low-carbon energy consumption [1]. According to China's energy development strategic plan and natural gas transport pipeline network plan, the share of natural gas in the primary energy consumption will continue to increase up to $10 \%$ by 2020 , while the total length of natural gas pipelines is planned to reach $104,000 \mathrm{~km}$. Driven by the continued growth of consumption and infrastructure strengthening, the trunk pipeline coverage will be further expanded and the regional gas transport pipeline network will be improved.

The natural gas transport pipeline network is tasked with gas distribution, which plays a significant role in improving the overall regional socioeconomic conditions. Although pipeline projects require a large investment and have a long payback period, the gas market is exhibiting a dynamic development trend [2]. Therefore, when planning the pipeline network, the benefits from investments on pipeline projects, construction costs, other influential factors, and the impact of uncertainty should be considered to determine the optimal layout scheme.

Previous studies on the layout of natural gas transport pipeline networks have proposed several methods based on pipeline network topology, such as graph theory, dynamic programming, neural networks, genetic algorithms, and complex methods $[3,4]$. These methods essentially solve the network structure to satisfy a given criterion and focus on mathematical optimization $[5,6]$. The minimum spanning 
TABLE 1: Advantages and limitations of existing methods.

\begin{tabular}{|c|c|c|}
\hline Methods & Advantages & Limitations \\
\hline Graph Theory & $\begin{array}{l}\text { Easy access to computer program } \\
\text { processing; higher computing efficiency; } \\
\text { effectively solving the shortest tree problem } \\
\text { within a large scale network }\end{array}$ & $\begin{array}{c}\text { Only solves the network layout among known fixed } \\
\text { points; project investment costs are not considered; } \\
\text { solutions can only be used as the initial network } \\
\text { layout }\end{array}$ \\
\hline Dynamic Programming & $\begin{array}{l}\text { Solving optimization problems with } \\
\text { multiple decision-making variables }\end{array}$ & $\begin{array}{c}\text { Not suitable for dealing with large-scale network } \\
\text { systems, dimension obstacles exist in solution } \\
\text { process }\end{array}$ \\
\hline Neural Network Method & $\begin{array}{l}\text { Solving optimization problems with } \\
\text { multiple decision-making variables }\end{array}$ & Only obtaining the local optimal solution \\
\hline Genetic Algorithm & $\begin{array}{l}\text { Higher adaptability that can overcome the } \\
\text { difficulties of solving nonlinear optimization }\end{array}$ & $\begin{array}{l}\text { Lower computing efficiency; no effective quantitative } \\
\text { analysis concerning algorithm precision, feasibility } \\
\text { and computational complexity }\end{array}$ \\
\hline Complex Method & $\begin{array}{c}\text { The algorithm is simple and suitable for } \\
\text { dealing with constrained optimization } \\
\text { problems }\end{array}$ & $\begin{array}{l}\text { Unable to deal with multi-variable, multi-constraint } \\
\text { optimization problems }\end{array}$ \\
\hline
\end{tabular}

tree method (MSTM) and dynamic programming (DP) are the most commonly used solutions. MSTM abstracts the pipeline network into an undirected network, including the classic solutions of the Dijkstra, Kruskal, and Steiner algorithms $[7,8]$. Compared to traditional graph theory solutions, these algorithms are implemented by computer programs for processing and have a relatively higher operational efficiency. The Steiner algorithms are effective in solving the shortest path problem of a large-scale network [9-11]. However, the three algorithms mentioned above do not consider the investment costs of pipeline projects, and their results can only be regarded as the initial pipeline network layout. DP can deal with the optimization problem of multiple decision-making variables. However, dimension obstacles exist during the solution process. Specifically, the computation will increase exponentially as the number of variables grows. When the dimension of this problem increases to a certain extent, the problem cannot be solved [12]. Thus, the current commonly used methods have certain advantages and limitations, as presented in Table 1. In summary, the optimization of the natural gas transport pipeline network is a multiobjective nonlinear programming problem, which should consider the uncertainty caused by the gas market and costs. However, this problem cannot be solved easily and effectively by using the abovementioned methods.

With regard to uncertainty and network layout, previous studies have mainly focused on transportation and logistics [13-15]. For example, regarding uncertainty and traffic network layout, Partriksson used a stochastic bilevel programming model to solve the optimal transportation network layout scheme based on the uncertainty of demand [16]. Yin et al. [17, 18] studied the urban road network layout methods under the impact of demand uncertainty and proposed sensitivity-based, scenario-based, and minmax optimization models. Zhang et al. [19] investigated the joint optimization problem of the green vehicle scheduling and routing problem in time-varying traffic networks and developed a corresponding joint optimization model. However, extensive studies on uncertainty associated with and the layout of the natural gas transport pipeline network have not been reported.

Therefore, this study uses the dominance degree model (DDM) of pipeline projects and the corresponding layout method, which considers socioeconomic benefits and construction costs. The layout method based on the DDM is simple: it provides a global optimal solution to obtain the comprehensive benefits of the pipeline network. Therefore, by analyzing the uncertain influential factors of the DDM, this study proposes an innovative uncertainty analysis of the natural gas transport pipeline network layout based on the Monte Carlo method. This proposed method uses the Monte Carlo method and sensitivity analysis to determine the impact of uncertainty factors on the model results. This can quantify the uncertainty and its influence and thus strengthen the practicability of the DDM and function as a future reference for the optimal layout of the pipeline network. Finally, to verify the validity of the methodology, natural gas transport pipeline projects in Zhejiang Province, China, are taken as a case study.

\section{Dominance Degree Model and Its Layout Method}

The dominance degree model (DDM) of pipeline projects is a new method that was developed to optimize the pipeline network layout [20]. This method used the dominance degree to reflect the comprehensive benefits of transport pipeline projects by combining the potential model (PM) and economic potential theory (EPT) to build the dominance degree model (DDM) for pipeline projects.

The DDM of pipeline projects embodies the comprehensive socioeconomic benefits of the projects. By comparing the dominance degree of pipeline projects when applying the DDM to the layout of natural gas transport pipeline network, the optimal layout scheme and construction sequences are determined, which will leverage the advantages of pipeline projects to obtain the maximum socioeconomic benefits of the pipeline network. 
TABLE 2: Calibration for the value of $K_{i}$.

\begin{tabular}{lc}
\hline$P(10,000$ People $)$ & $K_{i}$ \\
\hline$<20$ & 1 \\
$20 \sim 50$ & 1.25 \\
$50 \sim 100$ & 1.5 \\
$100 \sim 300$ & 2.0 \\
$300 \sim 500$ & 2.5 \\
$500 \sim 1000$ & 3.0 \\
\hline
\end{tabular}

2.1. Dominance Degree Model. This study combined the potential model (PM) and economic potential theory (EPT) to establish the dominance degree model (DDM) of the pipeline projects. The following assumptions and methods were used when developing the model:

(1) Pipeline projects will generate economic potential, which can be regarded as an index reflecting the city scale.

(2) Construction costs of pipeline projects will directly affect their economic benefits; therefore, construction costs were taken as a parameter reflecting the impedance in the PM.

(3) The influence of pipeline projects on economic potential of the city is closely related to its development level. Pipeline projects will directly affect the transformation of economic potential.

Therefore, some specific indicators can be selected to reflect these influential factors. Above all, the DDM of gas transport pipeline projects is established as follows:

$$
A_{i j}=K_{i} \frac{\mu_{i} I_{i}}{V G \beta a_{i j}^{2}+a_{i j} \sum_{k=1}^{4} m_{k} w_{k}},
$$

where $A_{i j}$ is the dominance degree of pipeline projects between city $i$ and gas transport station $j . K_{i}$ is the dielectric constant, which is closely related to the urban functional orientation of cities and towns. For the DDM of natural gas transport pipeline projects, the city scale $P$ is selected to reflect this parameter. Considering the adjustment in 2014 of the classification criteria for city scales, $K_{i}$ can be calibrated as shown in Table 2.

$\mu_{i}$ is related to the richness of urban resources, market development level, living standards of residents, and existing state of the industry. In this study, the macroeconomic indicator of GDP per capita is used to represent this parameter.

$I_{i}$ is the gas demand of city $i$, which is related to the local energy consumption structure and policies. To facilitate the analysis of this question, the energy consumption per unit GDP is selected to reflect the status of energy consumption and saving. To some extent, it also reflects the current gas demand and development potential of natural gas.

$a_{i j}$ is the distance between city $i$ and gas transport station $j$, which can be expressed by a straight-line distance.

$V$ is the pipeline price per unit weight. $G$ is the pipeline weight per unit length. The values of $V$ and $G$ can be obtained from pipelines of the same diameter, material, and thickness, as a standard. $\beta$ is the terrain correction coefficient, which is used to modify the pipeline route length. Different topography conditions have different values, which can be determined by referring to engineering data within the study area. $m_{k}$ is the number of crossings of the $k$ th type, and $w_{k}$ is the corresponding construction cost. $k=1,2,3,4$ represent a river, grade highway, substandard highway, and railway, respectively.

2.2. Layout Methods Based on DDM. By analyzing and comparing the dominance degree of pipeline projects within the natural gas transport pipeline network, the layout methods based on DDM can not only determine the optimal layout scheme, but also obtain construction sequences of pipeline projects. Thus, the socioeconomic benefits of the pipeline network will be maximized. The basic application process is shown in Figure 1.

According to the layout process, the layout method for a natural gas transport network based on DDM consists of four steps, as follows.

Step 1 (abstract pipeline network in the regional area). First, abstract gas transport stations and valve chests into spatial nodes and record a set of nodes $j=\{1,2, \ldots, m\}$. Determine their locations on the map by using a geographic information system (GIS). Then, connect these nodes based on pipeline alignments and obtain a spatial structure diagram of the regional pipeline network.

Step 2 (calculate the dominance degree of pipeline projects). Similarly, abstract the cities and towns uncovered by the gas transport pipeline network into a set of nodes $i=\{1,2, \ldots, n\}$. Calibrate model parameters and use the DDM to calculate the dominance degree between node $i$ and node $j$ sequentially. Then, sort the calculation results in descending order.

Step 3 (update the pipeline network in the regional area). According to the calculation results, take the pipeline project of maximum dominance degree and connect its nodes at both ends to form a new pipeline route. Then, the corresponding nodes $i$ are incorporated into a set of node $j$ : that is, $j=$ $\{1,2, \ldots, m+1\}$. For example, assume that $i=\{1,2,3,4\}$ and $j=\{1,2\}$, the calculated maximum dominance degree $A_{21}$, which means $i=2$ is removed from a set of node $i$, subsumed into a set of node $j$ and denoted as $j=3$; then, $i=\{1,3,4\}$ and $j=\{1,2,3\}$. Finally, the updated spatial structure diagram of the regional pipeline network is obtained.

Step 4 (calculate cyclically and output the results). Repeat Steps 2 and 3 until the set of nodes $i=\{1,2, \ldots, n\}$ is an empty set, while the set of nodes $j=\{1,2, \ldots, m+n\}$. At this time, all cities and towns have been covered by the pipeline network and the layout scheme of pipeline network is obtained.

\section{Uncertainty of Dominance Degree Model}

3.1. Source of Model Uncertainty. The uncertainty of the DDM arises from a series of factors, including wrong settings of the model, imperfect input information, and the inherent randomness of events and behaviors [21]. The PM and EPT are combined to establish the DDM, which fully embodies the comprehensive socioeconomic benefits of pipeline projects. 


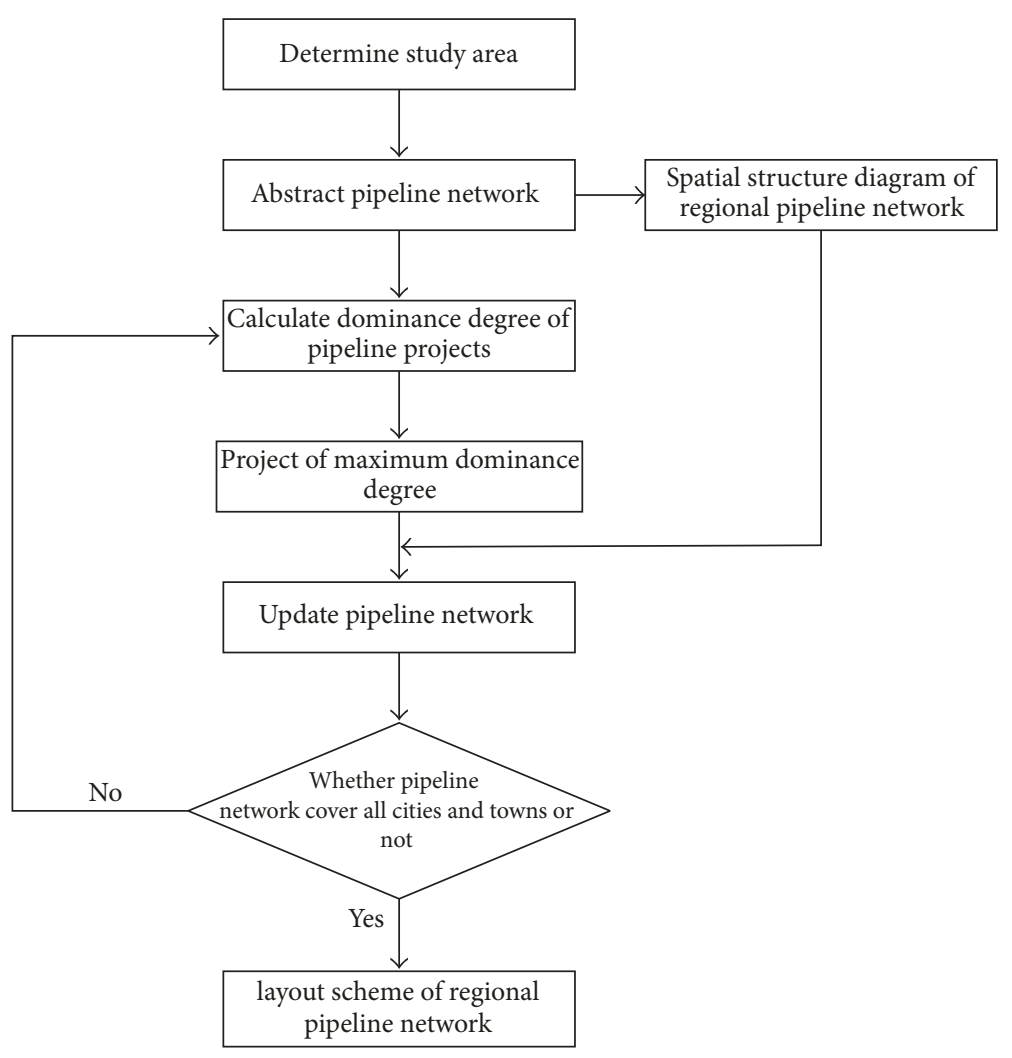

FIGURE 1: Natural gas transport pipeline network layout process.

However, the natural gas market is subject to economic development, gas pricing, policy adjustments, and other factors, whose development trend shows a certain irregularity. In addition, the construction costs of pipeline projects vary when the market prices and construction costs of steel pipelines change. When applying the DDM to obtain the layout scheme of a pipeline network, the inputs that reflect the market scale and construction costs should be determined based on statistical data or similar empirical engineering data. Therefore, there is a certain degree of uncertainty in the DDM, which is mainly derived from the inputs.

3.2. Uncertainty of Inputs. Model inputs mainly refer to data that describes the basic situation and related events and factors that affect the model performance [22]. The inputs of the DDM are obtained through related statistical survey data and by reference to similar empirical engineering data, which have a certain uncertainty. The survey data are the basic data, which are mainly used to characterize the urban socioeconomic conditions, energy consumption, and related policies along the pipeline project. Generally, there are errors in the surveys, although such data errors are somewhat limited to a certain extent and do not expand in the layout loop computation thanks to modern statistical methods. Therefore, the impact of the survey data error on the model uncertainty is relatively small. However, the inputs determined by similar empirical engineering data, such as the terrain correction coefficient and construction costs, will have a significant impact on model uncertainty. If, owing to conditional constraints, the survey of geographical environment conditions along the pipeline project is not detailed, the data will be more subjective. There are inevitable differences in geographical and environmental conditions along different natural gas transport pipeline projects, and thus, analogous data from similar projects may differ from the actual project. Therefore, the uncertainty of the model is likely to be dominated by the uncertainty of these inputs.

\section{Uncertainty Analysis of Model}

4.1. Quantitative Analysis of Uncertainty. The method of moments and Monte Carlo method are two main methods used to evaluate the model outputs [23]. The method of moments requires that the outputs are specified as an explicit, single function of the inputs, where higher order derivatives are used to ensure computational accuracy [24]. From the perspective of model setting, owing to the computational complexity inherent to the DDM and its layout method, it is difficult to calculate the outputs by the method of moments. Therefore, this study uses the Monte Carlo method, since it can obtain more accurate results to quantify the uncertainty of the DDM. According to the general process of Monte Carlo method [25], based on the characteristics of the DDM, the quantitative analysis of uncertainty includes the following three aspects. 
4.1.1. Determine the Probability Distribution of Inputs. The appropriate probability distribution is selected based on the characteristics of inputs of the DDM. In the DDM, many variables vary within the nonnegative range. To avoid the occurrence of negative numbers in the process of generating random numbers, the logarithmic normal distribution (LND) is used to represent the probability distribution of the DDM. The probability density function of the LND can be calculated as follows:

$$
f(t)=\frac{1}{\sqrt{2 \pi} \sigma_{\text {Int }}} \exp \left[-\frac{1}{2}\left(\frac{\text { Int }-\mu_{\text {Int }}}{\sigma_{\text {Int }}}\right)^{2}\right],
$$

where $\mu_{\text {Int }}$ is the location parameter, which represents the logarithmic mean of the probability distribution. $\sigma_{\text {Int }}$ is the shape parameter, which represents the logarithmic standard deviation.

The mean and variance of random variable $t$ can be represented as follows:

$$
\begin{aligned}
& E(t)=\exp \left(\mu_{\text {Int }}+\frac{1}{2} \sigma_{\text {Int }}^{2}\right) \\
& D(t)=\left(e^{\sigma_{\text {Int }}^{2}}-1\right) \exp \left(2 \mu_{\text {Int }}+\sigma_{\text {Int }}^{2}\right) .
\end{aligned}
$$

From (3) and (4), the coefficient of variation $(\mathrm{CoV})$ can be obtained as follows:

$$
\begin{aligned}
\operatorname{CoV} & =\frac{\sqrt{D(t)}}{E(t)}=\frac{\sqrt{\left(e^{\sigma_{\text {Int }}^{2}}-1\right) \exp \left(2 \mu_{\text {Int }}+\sigma_{\text {Int }}^{2}\right)}}{\exp \left(\mu_{\text {Int }}+(1 / 2) \sigma_{\text {Int }}^{2}\right)} \\
& =\sqrt{e^{\sigma_{\text {Int }}^{2}-1}} .
\end{aligned}
$$

4.1.2. Determine the CoV of Inputs. The $\mathrm{CoV}$ can be used to compare the degree of variation among two or more units [26]. Therefore, the $\mathrm{CoV}$ can be regarded as the expression of parameters of uncertainty. Considering that the $\mathrm{CoV}$ of some inputs is difficult to be determined directly, the CoV of inputs can be assumed based on the relevant research results to analyze its impact on the outputs of the DDM [27]. Moreover, the standard deviation can be calculated by multiplying the $\mathrm{CoV}$ and mean value of the inputs.

4.1.3. Simulation Analysis of Dominance Degree Model. The MATLAB software was used to program and solve the Monte Carlo simulation analysis of the DDM. According to the probability distribution of inputs, random samples are generated. Then, the results of outputs of DDM and their distribution can be calculated.

4.2. The Analysis Method of Uncertainty. This study uses a multivariate sensitivity analysis method to quantify the uncertainty of the DDM. Based on the linear regression of inputs and outputs, the impact of inputs on output uncertainty is analyzed. This method provides assistance in determining this impact and uses the normalized regression coefficient to represent the influence degree $[28,29]$.
4.2.1. Multiple Linear Regression Analysis. The multiple linear regression analysis is a statistical method to investigate the relationship between a dependent variable and multiple independent variables [30]. Assume that there is a number of independent variables $x_{1}, x_{2}, \ldots, x_{p}$ and a dependent variable $Y$. The model between them can be expressed as follows:

$$
Y=\beta_{0}+\beta_{1} x_{1}+\beta_{2} x_{2}+\cdots+\beta_{p} x_{p}+\varepsilon,
$$

where $\beta_{0}, \beta_{1}, \ldots, \beta_{p}$ represents the regression coefficient. $\varepsilon$ represents the error term that obeys normal distribution $N\left(0, \sigma^{2}\right)$.

By observing the variables $x_{1}, x_{2}, \ldots, x_{p}, Y$ for $n$ times, the observations of $\left(x_{i 1}, x_{i 2}, \ldots, x_{i p}, y_{i}\right)$ of $n$ groups are obtained. The least squares method is used to calculate $\beta_{0}, \beta_{1}, \ldots, \beta_{p}$, and then, the estimated value $\widehat{\beta}_{0}, \widehat{\beta}_{1}, \ldots, \widehat{\beta}_{p}$ can be obtained.

When carrying out the model uncertainty analysis, the outputs are set as dependent variables and the inputs are set as independent variables. Combined with the Monte Carlo simulation results, the regression coefficients were obtained by regression analysis.

4.2.2. Significance Tests of Regression Coefficients. To test if a regression coefficient $\beta_{j}(1 \leq j \leq p)$ is zero, it is equivalent to test whether its corresponding value $x_{j}$ has an impact on $Y$. Based on the linear regression model, we assume that $H_{0}$ : $\beta_{j}=0,1 \leq j \leq p$, and use $\widehat{\beta}_{j}$ as a test to obtain the statistics $T$ of Student's distribution.

$$
\begin{aligned}
T & =\frac{\widehat{\beta}_{j}}{\sqrt{c_{j j}} \sqrt{Q /(n-p-1)}} \\
Q & =\sum_{i=1}^{n}\left(Y_{i}-\widehat{y}_{i}\right)^{2},
\end{aligned}
$$

where $c_{j j}$ represents the $j$ th element on the main diagonal of matrix $C=A^{-1}$ and matrix $A$ is expressed as follows:

$$
A=\left[\begin{array}{ccccc}
n_{i} & \sum_{i} x_{i 1} & \sum_{i} x_{i 2} & \cdots & \sum_{i} x_{i p} \\
\sum_{i} x_{i 1} & \sum_{i} x_{i 1}^{2} & \sum_{i} x_{i 1} x_{i 2} & \cdots & \sum_{i}^{i} x_{i 1} x_{i p} \\
\vdots & \vdots & \vdots & & \vdots \\
\sum_{i} x_{i p} & \sum_{i} x_{i p} x_{i 1} & \sum_{i} x_{i p} x_{i 2} & \cdots & \sum_{i} x_{i p}^{2}
\end{array}\right] .
$$

Given a significant level $\alpha$, if $|T| \geq t_{\alpha / 2}(n-p-1)$, $H_{0}$ is rejected, and $\beta_{j}$ is significantly not equal to zero. If $|T|<t_{\alpha / 2}(n-p-1), H_{0}$ is accepted, and $\beta_{j}$ is significantly equal to zero. When making a significant test of regression coefficients, the value of $T$ and $p$ of $\beta_{j}$ can be obtained. If $p<\alpha$, it can also be determined that it is not equal to zero; namely, $x_{j}$ has a significant impact on model outputs. If $p>\alpha, x_{j}$ has no impact on model outputs, and thus, the variable can be excluded. 


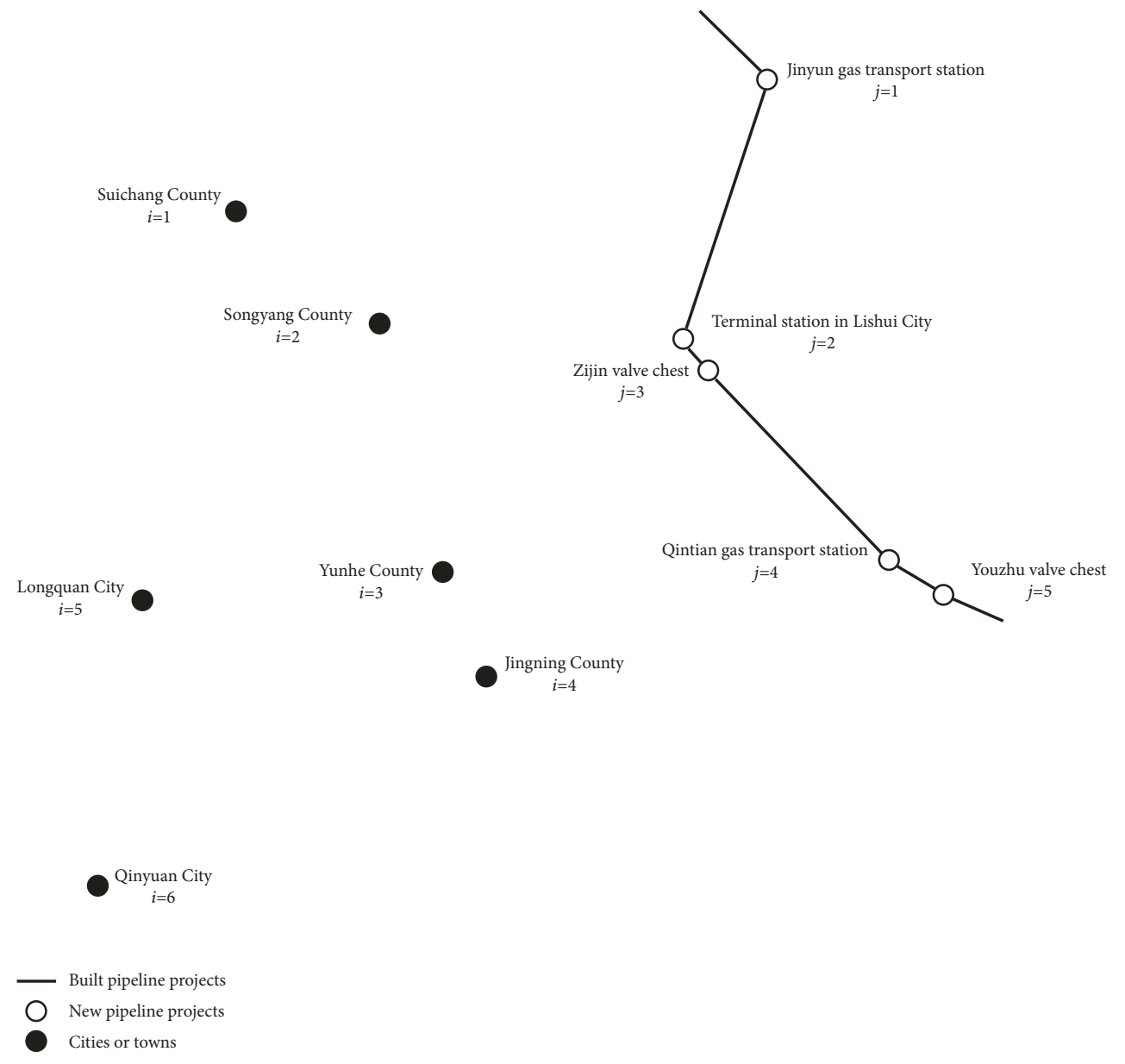

FIGURE 2: Natural gas transport pipeline network.

4.2.3. Sensitivity Analysis. A nonparametric statistics method is proposed to examine sensitivity [31]. After the linear regression analysis and significance test of model outputs and inputs, the standardized regression coefficient $\mathrm{SRC}_{i}$, which can be used to determine the impact of inputs on the uncertainty of outputs, can be calculated as follows:

$$
\mathrm{SRC}_{i}=\frac{\beta_{i} \times \sigma_{i}}{\sigma_{y}},
$$

where $\beta_{i}$ represents the regression coefficient. $\sigma_{i}$ represents the standard deviation of independent variables. $\sigma_{y}$ represents the standard deviation of dependent variables.

\section{Case Study}

The Lishui region along the Jinhua-Lishui-Wenzhou gas transport trunk pipeline engineering in Zhejiang Province, China, is taken as a case study. DDM and uncertainty analysis are used to determine the optimal layout scheme.

5.1. Pipeline Network and Model Inputs. GIS is used to determine the locations of the three natural gas transport stations and two valve chests. Then, the pipeline network in the regional area is schematized as shown in Figure 2.

According to the Statistical Yearbook of Lishui City of 2015, and energy consumption statistics of each county of Lishui City (Mátyás 1998), input parameters are as listed in Table 3.

By reference to related data of the Lishui section of the Jinhua-Lishui-Wenzhou pipeline engineering project, the L450M steel is used for standard pipelines. The value of $\beta$ is 1.032. $V$ is 0.77 ten thousand yuan/tons. $G$ is 222.3 tons $/ \mathrm{km}$. $w_{1}, w_{2}, w_{3}, w_{4}$ are, respectively, $250,15,4$, and 25 thousand yuan/crossing.

5.2. Model Calculation Results. By assuming that the model inputs obey the LND, the CoV is assigned a value of 0.3 based on related studies $[32,33]$. Using the proposed methodology, the two layout schemes and project construction sequence of the gas pipeline network in the Lishui area are obtained, as shown in Figures 3 and 4.

The results present in Table 4 are obtained by setting the significant level $\alpha=0.05$ and using the multivariate sensitivity analysis method to quantify uncertainty of the DDM. 


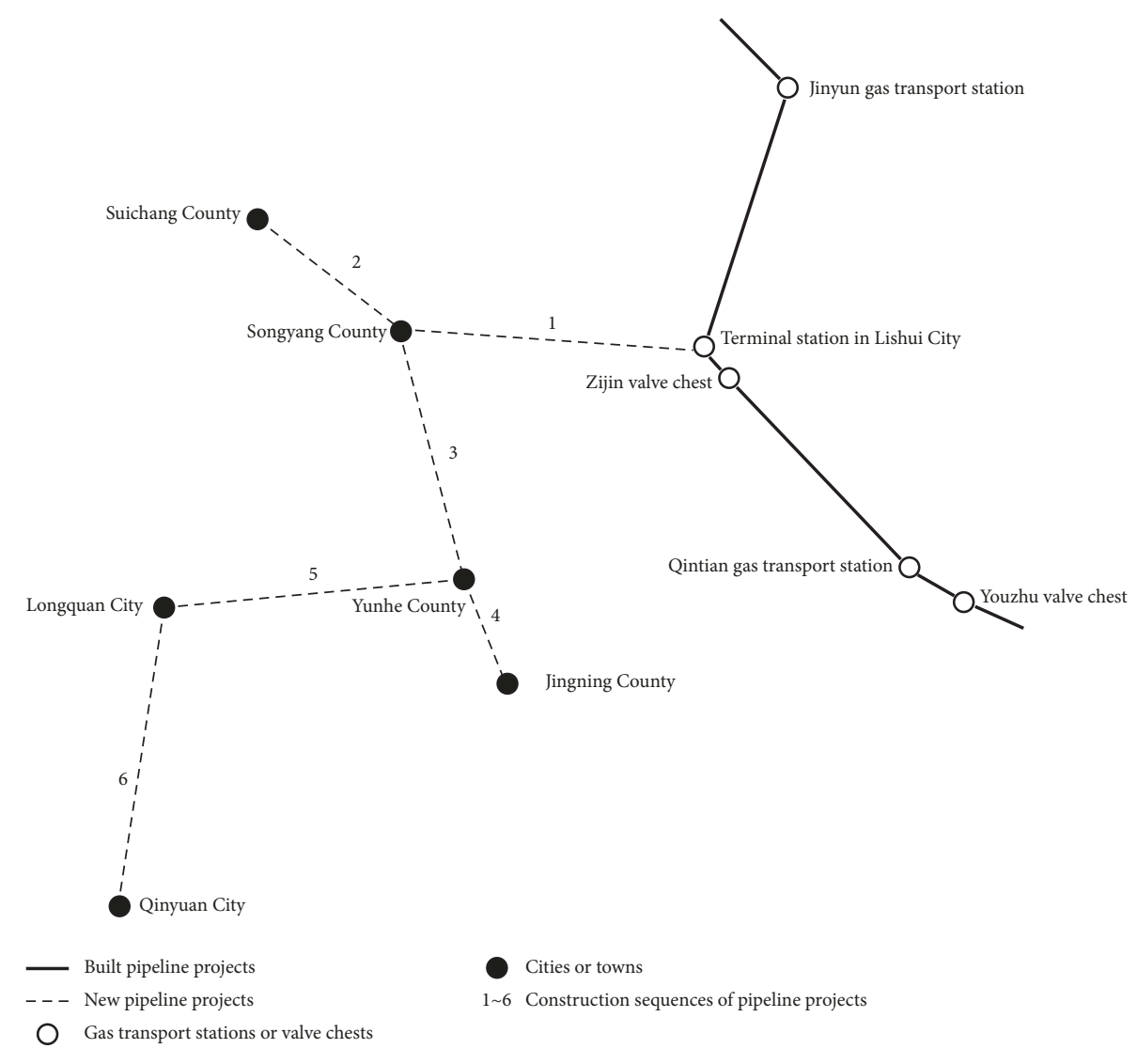

FIGURE 3: Layout scheme of natural gas transport pipeline network (Scheme 1).

TABLE 3: Input parameters of cities and towns.

\begin{tabular}{lccc}
\hline Name & $K$ & $M(10,000$ yuan/person) & $I$ (Tons of standard coal/10,000 yuan) \\
\hline Suiyang County & 1.25 & 46.596 & 0.470 \\
Songyang County & 1.25 & 44.037 & 0.456 \\
Yunhe County & 1 & 45.627 & 0.532 \\
Jingning County & 1 & 38.541 & 0.458 \\
Longquan City & 1.25 & 39.546 & 0.491 \\
Qinyuan County & 1.25 & 43.943 & 0.490 \\
\hline
\end{tabular}

TABLE 4: Results of sensitivity analysis.

\begin{tabular}{lcc}
\hline Inputs & SRC & $A$ \\
\hline$\mu$ & 3.050 & $P$ \\
$K$ & 1.739 & 0.000 \\
$I$ & 0.379 & 0.001 \\
$a$ & -3.326 & 0.030 \\
$w_{1}$ & -1.594 & 0.000 \\
$w_{4}$ & -0.750 & 0.000 \\
$w_{2}$ & -0.279 & 0.001 \\
$\beta$ & -0.221 & 0.001 \\
$V$ & -0.167 & 0.025 \\
\hline
\end{tabular}

The results reveal that the uncertainty of model inputs directly affects the pipeline network layout schemes and optimal construction sequences. The inputs having a significant impact on the uncertainty of the DDM are the GDP per capita $\mu$, the dielectric constant $K$, the distance between city and gas transport station $a$, and the corresponding construction costs of crossing a river and railway $w_{1}, w_{4}$.

Natural gas consumption and city scale are closely related to economic development level, which determine the market development potential and growth rate and become the main influential factors that affect the natural gas market. Thus, the uncertainty of $\mu$ and $K$ has a significant impact on model results. The pipeline project construction cost is related to the length of pipelines $a$ and the corresponding construction costs of crossings, which have a direct impact on the project 


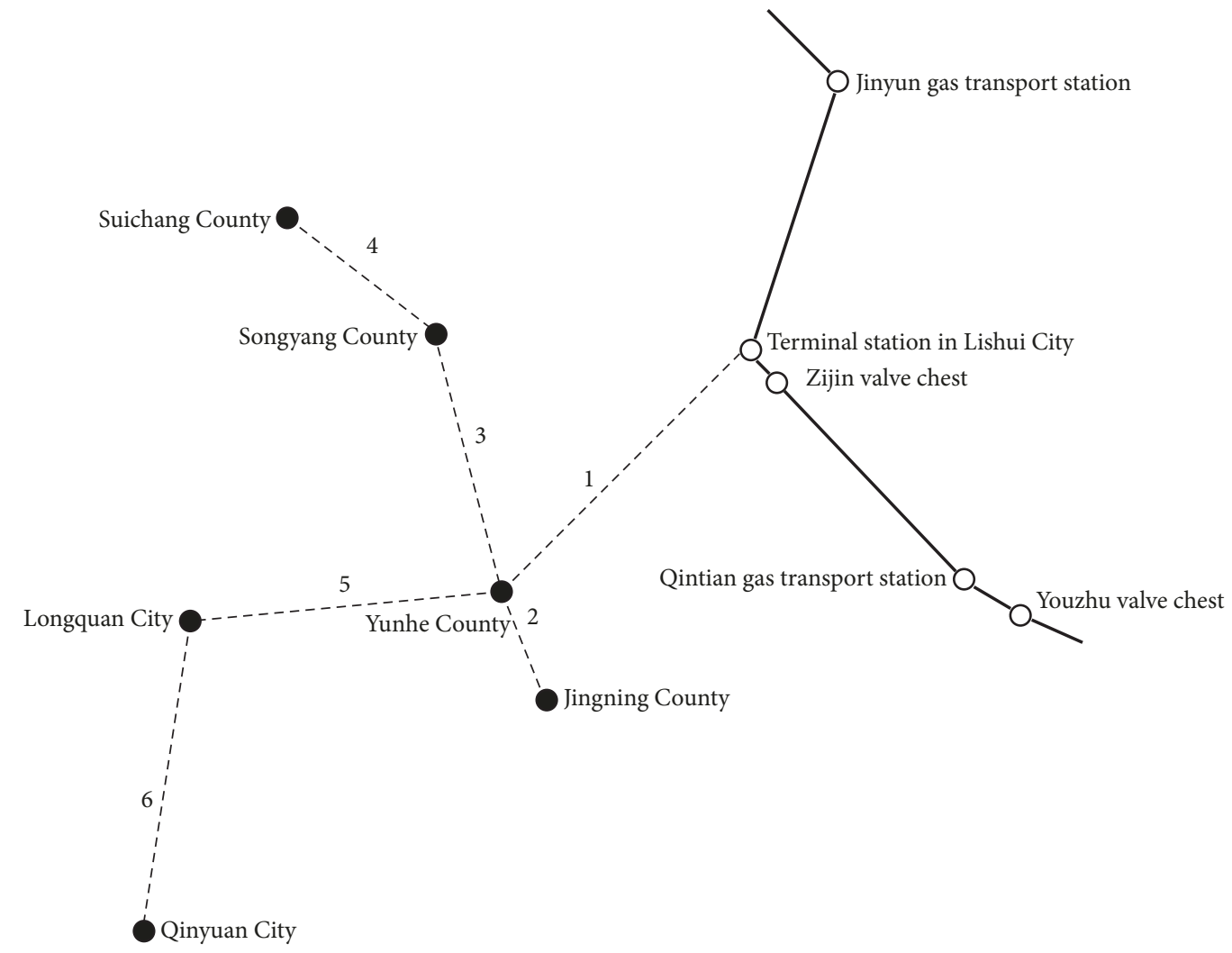

- Built pipeline projects
-- New pipeline projects
Gas transport stations or valve chests

- Cities or towns

1 6 Construction sequences of pipeline projects

FIGURE 4: Layout scheme of natural gas transport pipeline network (Scheme 2).

investment and income. The higher costs of $w_{1}$ and $w_{4}$ directly affect construction costs. Thus, the uncertainty of $a, w_{1}$, and $w_{4}$ has a significant impact on the results of the model.

In summary, when the DDM is applied to determine the layout of a natural gas transport pipeline network, we should consider the impact of input uncertainty, so as to try and reduce this impact and improve the accuracy of the model results. Accordingly, this will support the best decision regarding the layout of the natural gas transport pipeline network.

\section{Conclusions and Future Studies}

The optimization of the layout of a natural gas transport pipeline network is a scientific and forward-looking topic with socioeconomic benefits. It is also a complex problem, which is affected by multiple factors including uncertainty. This study investigated the DDM and its layout method and analyzes the source and impact of model uncertainty and then proposed a new methodology for uncertainty analysis based on the Monte Carlo method. The Jinhua-Lishui-Wenzhou gas transport trunk pipeline engineering project at the Lishui region in Zhejiang Province, China, was taken as a case study, and the corresponding pipeline network layout scheme and the uncertainty analysis results were obtained.

Uncertainty is one type of limitation that exists almost in every model. The uncertainty analysis method that was developed for the DDM is a method that can quantify the uncertainty of the model and examine the influence of different uncertainty factors on the layout of a regional pipeline network. It was observed from the case study results that the uncertainty of the model directly affected the layout optimization for the gas transport pipeline network and provided us with different results. Therefore, in practical applications of the DDM for regional network layouts, it is necessary to conduct an uncertainty analysis and quantify uncertainty effects of the model. Through the uncertainty analysis, we were able to determine the factors that significantly affect the model uncertainty and take measures to improve the accuracy of these factors by reducing the influence of uncertainty and obtaining the optimal layout of gas transport pipeline network, which could be used as a reference and provide technical support to decision-making with regard to an optimal pipeline network layout.

However, there are several limitations in the present study. First, this study set the CoV to reflect changes in model inputs, while various differences existed between the set 
based on the empirical data and the actual situation. Thus, the method to determine the value of the CoV should be further studied. Second, a multivariate sensitivity analysis was used to analyze the impact of specific inputs on model uncertainty, while in reality, the model uncertainty may be caused by the combined impact of multiple inputs. Therefore, the impact of uncertainty derived from combinations of multiple inputs on the model results should be further studied.

\section{Conflicts of Interest}

The authors declare that there are no conflicts of interest regarding the publication of this paper.

\section{References}

[1] M. F. Chávez-Rodríguez, L. Dias, S. Simoes et al., "Modelling the natural gas dynamics in the Southern Cone of Latin America," Applied Energy, vol. 201, pp. 219-239, 2017.

[2] X. Ma and Z. Liu, "Application of a novel time-delayed polynomial grey model to predict the natural gas consumption in China," Journal of Computational and Applied Mathematics, vol. 324, pp. 17-24, 2017.

[3] S. Sanaye and J. Mahmoudimehr, "Optimal design of a natural gas transmission network layout," Chemical Engineering Research and Design, vol. 91, no. 12, pp. 2465-2476, 2013.

[4] H. Üster and Ş. Dilaveroğlu, "Optimization for design and operation of natural gas transmission networks," Applied Energy, vol. 133, pp. 56-69, 2014.

[5] R. R. Singh and P. K. S. Nain, "Optimization of natural pipeline design and its total cost using GA," International Journal of Science and Research Publications, vol. 2, no. 8, pp. 1-10, 2012.

[6] F. D. S. Alves, J. N. M. D. Souza, and A. L. H. Costa, "Multiobjective design optimization of natural gas transmission networks," Computers \& Chemical Engineering, vol. 93, pp. 212-220, 2016.

[7] J. An and S. Peng, "Layout optimization of natural gas network planning: Synchronizing minimum risk loss with total cost," Journal of Natural Gas Science and Engineering, vol. 33, pp. 255263, 2016.

[8] Z. Shariat, A. Movaghar, and M. Hoseinzadeh, "A learning automata and clustering-based routing protocol for named data networking," Telecommunication Systems, vol. 65, no. 1, pp. 929, 2017.

[9] Y. Cuan and X. Chen, "Application of improved Dijkstra algorithm in selection of gas source node in gas network," in Proceedings of the 2012 International Conference on Industrial Control and Electronics Engineering, ICICEE 2012, pp. 15581560, chn, August 2012.

[10] D.-M. Han and J.-H. Lim, "Design and implementation of smart home energy management systems based on ZigBee," IEEE Transactions on Consumer Electronics, vol. 56, no. 3, pp. 14171425, 2010.

[11] H. J. Prömel and A. Steger, The Steiner Tree Problem: A Tour through Graphs, Algorithms, And Complexity, Advanced Lectures in Mathematics, Friedr. Vieweg \& Sohn, Braunschweig, 2002.

[12] R. E. Bellman, S. E. Dreyfus, R. Bellman et al., Applied Dynamic Programming, Princeton University Press, Princeton, NJ, USA, 2015.
[13] T. M. Masaud, R. D. Mistry, and P. K. Sen, "Placement of large-scale utility-owned wind distributed generation based on probabilistic forecasting of line congestion," IET Renewable Power Generation, vol. 11, no. 7, pp. 979-986, 2017.

[14] M. M. J. Knoope, A. Ramírez, and A. P. C. Faaij, “The influence of uncertainty in the development of a $\mathrm{CO}_{2}$ infrastructure network," Applied Energy, vol. 158, pp. 332-347, 2015.

[15] X.-F. Xu, J. Hao, Y.-R. Deng, and Y. Wang, "Design optimization of resource combination for collaborative logistics network under uncertainty," Applied Soft Computing, vol. 56, pp. 684691, 2017.

[16] M. Patriksson, "Robust bi-level optimization models in transportation science," Philosophical Transactions of the Royal Society A: Mathematical, Physical and Engineering Sciences, vol. 366, no. 1872, pp. 1989-2004, 2008.

[17] Y. Yin, S. Lawphongpanich, and Y. Lou, "Estimating investment requirement for maintaining and improving highway systems," Transportation Research Part C: Emerging Technologies, vol. 16, no. 2, pp. 199-211, 2008.

[18] Y. Yin, S. M. Madanat, and X.-Y. Lu, "Robust improvement schemes for road networks under demand uncertainty," European Journal of Operational Research, vol. 198, no. 2, pp. 470479, 2009.

[19] D. Zhang, X. Wang, S. Li, N. Ni, Z. Zhang, and X. Ma, "Joint optimization of green vehicle scheduling and routing problem with time-varying speeds," PLOS ONE, vol. 13, no. 2, article e0192000, 2018.

[20] Z. Zhu, C. Sun, J. Zeng, and G. Chen, “Optimization of natural gas transport pipeline network layout: a new methodology based on dominance degree model," Transport, vol. 33, no. 1, pp. 143-150, 2018.

[21] K. Hirano and J. H. Wright, "Forecasting with model uncertainty: representations and risk reduction," Econometrica, vol. 85, no. 2, pp. 617-643, 2017.

[22] W. Xie, B. L. Nelson, and R. R. Barton, "Multivariate input uncertainty in output analysis for stochastic simulation," ACM Transactions on Modeling and Computer Simulation (TOMACS), vol. 27, no. 1, Art. 5, 22 pages, 2016.

[23] S. Mohajeryami and M. Doostan, "Investigating the lightning effect on compact transmission lines by employing Monte Carlo method," in Proceedings of the 2016 IEEE/PES Transmission and Distribution Conference and Exposition, May 2016.

[24] S. Lefrancois, C. Husko, A. Blanco-Redondo, and B. J. Eggleton, "Nonlinear silicon photonics analyzed with the moment method," Journal of the Optical Society of America B: Optical Physics, vol. 32, no. 2, pp. 218-226, 2015.

[25] R. Y. Rubinstein and D. P. Kroese, Simulation and the Monte Carlo Method, John Wiley \& Sons, Inc., 2008.

[26] W. C. Yeong, M. B. Khoo, S. L. Lim, and M. H. Lee, "A direct procedure for monitoring the coefficient of variation using a variable sample size scheme," Communications in Statistics-Simulation and Computation, vol. 46, no. 6, pp. 42104225, 2017.

[27] S.-P. Wan, "Multi-attribute decision making method based on possibility variance coefficient of triangular intuitionistic fuzzy numbers," International Journal of Uncertainty, Fuzziness and Knowledge-Based Systems, vol. 21, no. 2, pp. 223-243, 2013.

[28] E. Borgonovo and E. Plischke, "Sensitivity analysis: a review of recent advances," European Journal of Operational Research, vol. 248, no. 3, pp. 869-887, 2016. 
[29] M. Lamboni, H. Monod, and D. Makowski, "Multivariate sensitivity analysis to measure global contribution of input factors in dynamic models," Reliability Engineering \& System Safety, vol. 96, no. 4, pp. 450-459, 2011.

[30] G. Grégoire, "Multiple linear regression," EAS Publications Series, vol. 66, Article ID 1466005, pp. 45-72, 2015.

[31] A. Saltelli and J. Marivoet, "Non-parametric statistics in sensitivity analysis for model output: a comparison of selected techniques," Reliability Engineering \& System Safety, vol. 28, no. 2, pp. 229-253, 1990.

[32] W. L. Teoh, M. B. Khoo, P. Castagliola, W. C. Yeong, and S. Y. Teh, "Run-sum control charts for monitoring the coefficient of variation," European Journal of Operational Research, vol. 257, no. 1, pp. 144-158, 2017.

[33] M. E. Calzada and S. M. Scariano, "A synthetic control chart for the coefficient of variation," Journal of Statistical Computation and Simulation, vol. 83, no. 5, pp. 851-865, 2013. 


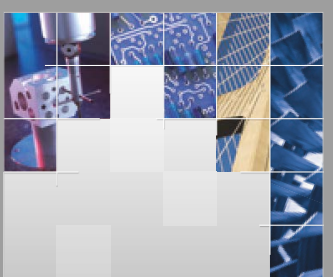

\section{Enfincering}
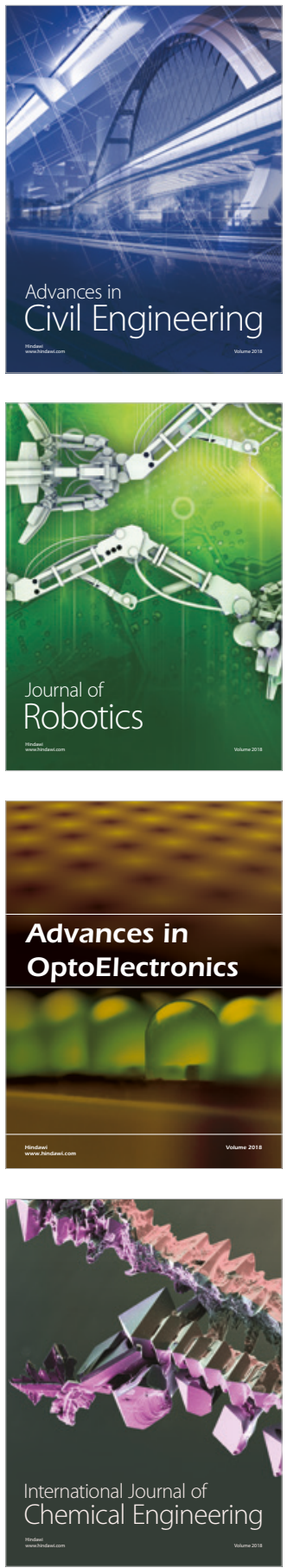

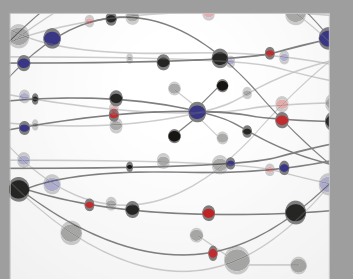

\section{Rotating \\ Machinery}

The Scientific World Journal

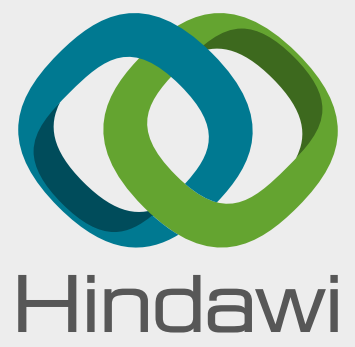

Submit your manuscripts at

www.hindawi.com
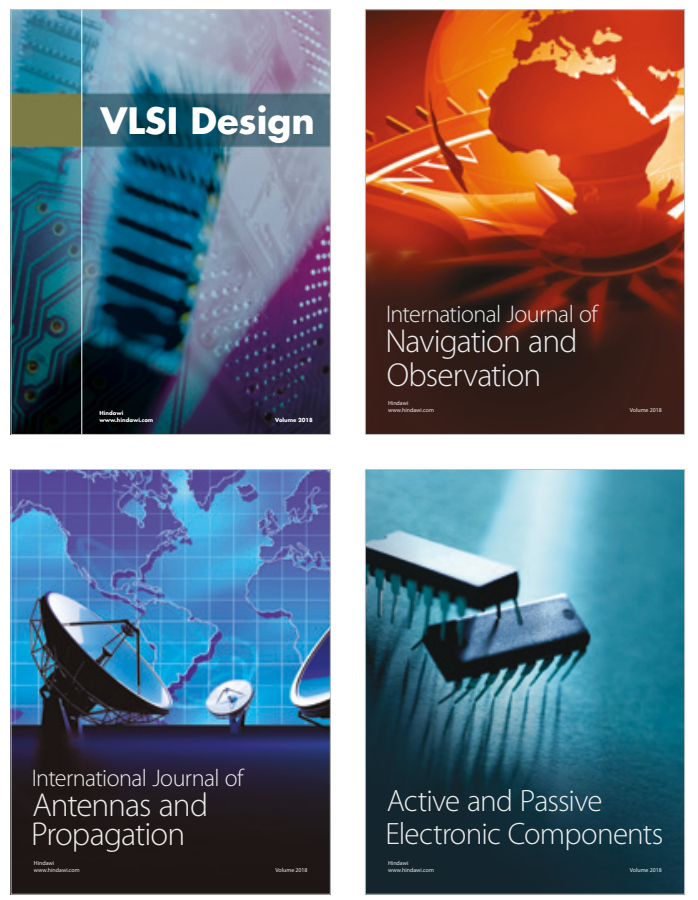
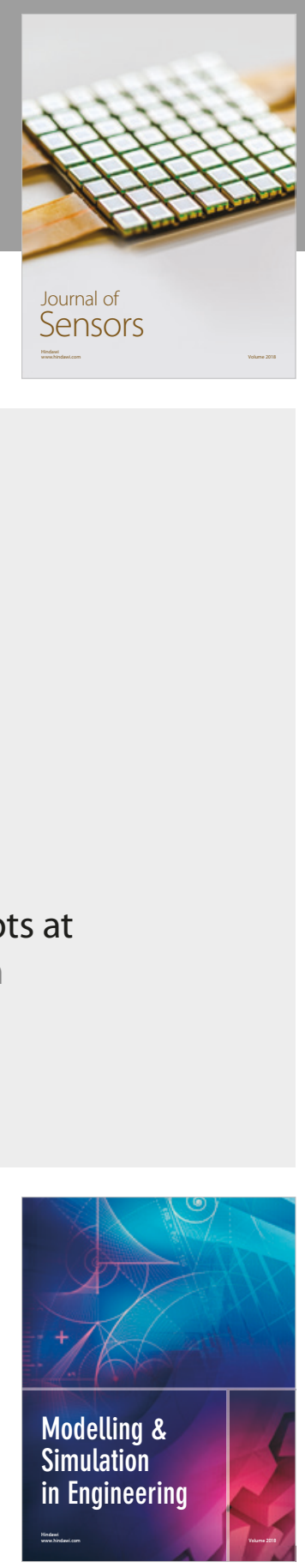

\section{Advances \\ Multimedia}
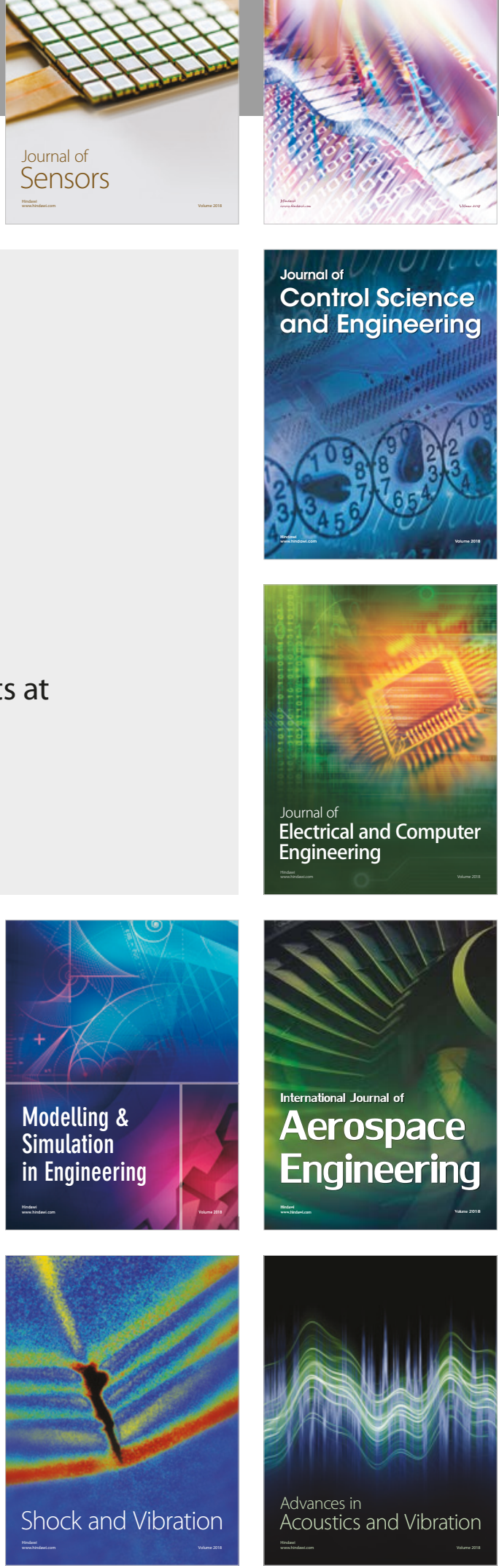